Table 1. Systematic list of Butterflies from Keoladeo National Park, Bharatpur, Rajasthan.

\section{ADDITIONS TO THE BUTTERFLIES OF KEOLADEO NATIONAL PARK, BHARATPUR, RAJASTHAN, INDIA.}

\author{
Muhamed Jafer Palot and V.P. Soniya \\ Zoological Survey of India, Freshwater Biological Station, 1-1-300/ \\ B, Ashok Nagar, Hyderabad, Andhra Pradesh 500020, India.
}

Previous field studies in the National Park revealed 34 species of butterflies in the 29sq.km. Keoladeo National Park, Bharatpur (Palot \& Soniya, 2000). Further, survey during the winter month of December, 1999 (1-7), added six more species to the list, making a total of 40 species from the Park. The notable addition to the list is the Common Crow (Euploea core), observed in large numbers along the road from Barrier (entry) to the Keoladeo Temple. Satyrids like Common Evening Brown (Melanitis leda) and Dark Brand Bushbrown (Mycalesis mineus) were also observed in fairly good numbers. Zebra Blue (Syntaurcus plinius) and Pointed Pierrot (Tarucus theophrastus) were the lycaenids added to the list. The Bank's Swift (Baoris kumara) was the only Hesperiid observed during the present survey. This species was observed to be associated with the nectary blue flowers of Hygrophila auriculata, a common spiny herb, found along the bund and trekpaths lining the wetland regions.

During winter, the activities of butterflies were more during the sunny hours and the population seemed to be apparently more confined to the sunlit areas of the Park. A notable feature was the absence of common species like Lemon Butterfly (Papilio demoleus), Little Orange Tip (Colotis etrida), Plain Orange Tip (Colotis eucharis), Crimson Tip (Colotis danae), Lemon Emigrant (Catopsilia pomona) and Common Castor (Ariadne merione), while, abundance of Common Crow (Euploea core), Blue Tiger (Tirumala limniace), Danaid Eggfly (Hypolimnas misippus) were noticed. A detailed systematic list with occurrence during summer survey (13-24 April, 1999) and winter survey (1-7 December, 1999) is given in Table 1.

\section{Acknowledgements}

We are thankful to Dr. J.R.B. Alfred, Director, ZSI and Dr. S.Z. Siddiqi, ZSI Hyderabad, for facilities and editing.

\section{Reference}

Palot, M.J. and V.P. Soniya (2000). Preliminary report on the butterflies of Keoladeo National Park, Bharatpur, Rajasthan, India. Zoos' Print Journal 15(6): 287-288.

\begin{tabular}{|c|c|c|c|}
\hline Scientific Name & Common Name & Apr. & Dec. \\
\hline \multicolumn{4}{|l|}{ Papilionidae } \\
\hline Pachliopta aristolochiae Fabricius & Common Rose & $P$ & $P$ \\
\hline Papilio polytes Linnaeus & Common Mormon & $\mathrm{P}$ & $\mathrm{P}$ \\
\hline Papilio demoleus Linnaeus & Lime Butterfly & $P$ & $A$ \\
\hline \multicolumn{4}{|l|}{ Pieridae } \\
\hline Leptosia nina Fabricius & Psyche & $P$ & $P$ \\
\hline Cepora nerissa Fabricius & Common Gull & $P$ & $P$ \\
\hline Anaphaeis aurota Fabricius & Caper White & $P$ & $P$ \\
\hline Colotis amata Fabricius & Small Salmon Arab & $P$ & $\mathrm{P}$ \\
\hline Colotis etrida Boisduval & Little Orange Tip & $P$ & $A$ \\
\hline Colotis eucharis Fabricius & Plain Orange Tip & $P$ & $A$ \\
\hline Colotis danae Fabricius & Crimson Tip & $P$ & $A$ \\
\hline Colotis vestalis Butler & White Arab & $P$ & $P$ \\
\hline Madais fausta Wallengren & Great Salmon Arab & $P$ & $P$ \\
\hline Ixias marianne Cramer & White Orange Tip & $P$ & $P$ \\
\hline Ixias pyrene Fabricius & Yellow Orange Tip & $\mathrm{P}$ & $P$ \\
\hline Catopsilia pomona Fabricius & Lemon Emigrant & $P$ & $A$ \\
\hline Catopsilia pyranthe Linnaeus & Mottled Emigrant & $P$ & $P$ \\
\hline Eurema brigitta Wallace & Small Grass Yellow & $P$ & $P$ \\
\hline Eurema hecabe Moore & Common Grass Yellow & $P$ & $P$ \\
\hline \multicolumn{4}{|l|}{ Danaidae } \\
\hline Danaus chrysippus Linnaeus & Plain Tiger & $P$ & $\mathrm{P}$ \\
\hline Danaus genutia Cramer & Striped Tiger & $P$ & $P$ \\
\hline Tirumala limniace Gmelin & Blue Tiger & $P$ & $P$ \\
\hline Euploea core Cramer & Common Crow & $A$ & $P$ \\
\hline \multicolumn{4}{|l|}{ Satyridae } \\
\hline Melanitis leda Drury & Common Evening Brown & $A$ & $P$ \\
\hline Mycalesis mineus Cramer & Dark Brand Bushbrown & $A$ & $P$ \\
\hline \multicolumn{4}{|l|}{ Lycaenidae } \\
\hline Azanus ubaldus Cramer & Bright Babul Blue & $P$ & $A$ \\
\hline Zizina otis Butler & Lesser Grass Blue & $\mathrm{P}$ & $P$ \\
\hline Zizula hylax Fabricius & Tiny Grass Blue & $P$ & $A$ \\
\hline Euchrysops cnejus Fabricius & Gram Blue & $P$ & $A$ \\
\hline Syntaurcus plinius Fabricius & Zebra Blue & $A$ & $P$ \\
\hline Tarucus theophrastus Fabricius & Pointed Pierrot & $A$ & $P$ \\
\hline \multicolumn{4}{|l|}{ Nymphalidae } \\
\hline Ariadne merione Cramer & Common Castor & $P$ & $A$ \\
\hline Phalantha phalantha Drury & Common Leopard & $\mathrm{P}$ & $P$ \\
\hline Junonia hierta Fabricius & Yellow Pansy & $P$ & $P$ \\
\hline Junonia orithya Butler & Blue Pansy & $P$ & $P$ \\
\hline Junonia lemonias Linnaeus & Lemon Pansy & $\mathrm{P}$ & $\mathrm{P}$ \\
\hline Junonia almana Linnaeus & Peacock Pansy & $P$ & $P$ \\
\hline Junonia atlites Linnaeus & Grey Pansy & $P$ & $P$ \\
\hline Hypolimnas misippus Linnaeus & Danaid Eggfly & $P$ & $P$ \\
\hline \multicolumn{4}{|l|}{ Hesperiidae } \\
\hline Suastus gremius Fabricius & Indian Palm Bob & $P$ & $A$ \\
\hline Baoris kumara Moore & Bank's Swift & $A$ & $P$ \\
\hline
\end{tabular}

P - Present; A - Absent. 